\title{
ON NUMBERS WHICH ARE DIFFERENCES OF TWO CONJUGATES OF AN ALGEBRAIC INTEGER
}

\author{
Artūras Dubickas
}

\begin{abstract}
We investigate which numbers are expressible as differences of two conjugate algebraic integers. Our first main result shows that a cubic, whose minimal polynomial over the field of rational numbers has the form $x^{3}+p x+q$, can be written in such a way if $p$ is divisible by 9 . We also prove that every root of an integer is a difference of two conjugate algebraic integers, and, more generally, so is every algebraic integer whose minimal polynomial is of the form $f\left(x^{e}\right)$ with an integer $e \geqslant 2$.
\end{abstract}

\section{INTRODUCTION}

Let $K$ be a number field, that is, a finite extension of the field of rational numbers $\mathbb{Q}$, and let $\mathbb{Z}_{K}$ be its ring of integers. (Recall that $a \in \mathbb{Z}_{K}$ if and only if $a \in K$ and its minimal polynomial over $\mathbb{Q}$, whose leading coefficient is equal to 1 , has all other coefficients lying in the ring of integers $\mathbb{Z}$.) Assume that $\beta$ is an algebraic number of degree $d$ over the field $K$ with conjugates $\beta_{1}=\beta, \beta_{2}, \ldots, \beta_{d}$.

QUESTION 1. Which numbers $\beta$ can be written as a difference $\alpha-\alpha^{\prime}$ of two conjugates over $K$ of an algebraic integer?

Recall that $\alpha$ is an algebraic integer if its minimal polynomial over $\mathbb{Q}$, whose leading coefficient is equal to 1 , has all other coefficients lying in the ring $\mathbb{Z}$. Then its minimal polynomial over $K$, whose leading coefficient is 1 , has all other coefficients in the ring $\mathbb{Z}_{K}$. Clearly, such $\beta$ itself must be an algebraic integer. Furthermore, $\beta$ must be expressible as a difference of two conjugates over $K$ of an algebraic number.

The set of numbers which are differences of two conjugates over $K$ was studied by the author and Smyth in [3]. It was shown that $\beta=\alpha-\alpha^{\prime}$ with some $\alpha$ and $\alpha^{\prime}$ conjugate over $K$ if and only if there is an automorphism $\sigma$ in the Galois group of $K\left(\beta_{1}, \ldots, \beta_{d}\right) / K$ of order $n$ such that $\sum_{i=0}^{n-1} \sigma^{i}(\beta)=0$. Then, setting $\alpha=\sum_{i=0}^{n-1}(n-i-1) \sigma^{i}(\beta) / n$, we indeed have $\beta=\alpha-\sigma(\alpha)$. (Compare with Hilbert's

Received 23rd October, 2001

I owe Question 1 to Chris Smyth. I would like to thank Andrzej Schinzel for his interest and a suggestion concerning the Cardano formulae. This research was partially supported by a grant from Lithuanian State Science and Studies Foundation.

Copyright Clearance Centre, Inc. Serial-fee code: 0004-9727/02 \$A2.00+0.00. 
Theorem 90 in its additive form. See, for example, [4] or [5, Chapter VIII, Section 6].) This implies that $\beta$ can only be expressible as above if its trace over $K$, namely, the sum of all its conjugates over $K$ is 0 .

Of course, the answer to Question 1 depends on $K$. For example, $\sqrt{2}$ is not expressible as a difference of two conjugates over $\mathbb{Q}(\sqrt{2})$ of an algebraic number. However it is a difference of two conjugates over $\mathbb{Q}$ of an algebraic integer, say, $\alpha=(\sqrt{6}+\sqrt{2}) / 2$ and $\alpha^{\prime}=(\sqrt{6}-\sqrt{2}) / 2$.

QUESTION 2. Is every algebraic integer which is expressible as a difference of two conjugates over $K$ of an algebraic number also expressible as a difference of two conjugates over $K$ of an algebraic integer?

The positive answer to Question 2 would immediately imply the following answer to Question 1: those $\beta$ which are algebraic integers and which are differences of two conjugates over $K$ of an algebraic number (the latter ones were described above).

\section{Results}

For $\beta$ of degree at most 3 , the condition on its trace is not only necessary, but also sufficient (see [3]). It follows that every $\beta$ of trace zero over $K$ and of degree at most 3 over $K$ is a difference of two conjugates over $K$. For $d=1$, only $\beta=0$ is of trace zero, and it is a difference of two zeroes. We begin with the following simple result.

OBSERVATION. Given a number field $K$, suppose that $\beta$ is an algebraic integer and $-\beta$ is its conjugate over $K$. Then $\beta$ can be written as a difference of two conjugates over $K$ of an algebraic integer.

Proof: Let $\beta$ be of degree $d$ over $K$. Choose $m \in \mathbb{Z}$ such that $\alpha=\beta / 2+$ $\sqrt{(\beta / 2)^{2}-m}$ is of degree $2 d$ over $K$. Then $\alpha$ is an algebraic integer, since so is $\beta$. Also, $-\beta / 2+\sqrt{(\beta / 2)^{2}-m}$ is conjugate to $\alpha$ over $K$. Their difference is $\beta$ which completes the proof.

The answers to the above questions for quadratic numbers now follow, since every quadratic number $\beta$ of trace 0 has $-\beta$ as its other conjugate.

COROLlary 1. For every number field $K$, a quadratic over $K$ algebraic integer $\beta$, whose minimal polynomial over $K$ is $x^{2}+p x+q$, can be written as a difference of two conjugates over $K$ of an algebraic integer if and only if $p=0$.

If, for instance, $K=\mathbb{Q}$ and $\beta=\sqrt{2}$, then, choosing $m=-1$, we have the above example with $\alpha=(\sqrt{6}+\sqrt{2}) / 2$ being the root of the irreducible over $\mathbb{Q}$ polynomial $x^{4}-4 x^{2}+1$. Can the real cubic root of 2 be expressed in a similar way?

Before we answer this question, consider the simplest case of cubics which are expressible as differences of conjugate algebraic integers. Let $\beta$ be a cubic algebraic 
integer over a number field $K$ of trace 0 with minimal polynomial $x^{3}+p x+q$ over $K$. Here, $p, q \in \mathbb{Z}_{K}$, and so does the discriminant of $\beta$,

$$
\Delta=\left(\left(\beta_{1}-\beta_{2}\right)\left(\beta_{1}-\beta_{3}\right)\left(\beta_{2}-\beta_{3}\right)\right)^{2}=-4 p^{3}-27 q^{2} .
$$

Set $\gamma=\gamma_{1}=\beta_{1}-\beta_{3}$. It has at least two other conjugates over $K, \gamma_{2}=\beta_{2}-\beta_{1}$ and $\gamma_{3}=\beta_{3}-\beta_{2}$, since the Galois group of $K\left(\beta_{1}, \beta_{2}, \beta_{3}\right) / K$ contains the 3-cycle (123). Clearly, every such $\beta$ of trace 0 is expressible as $\alpha-\alpha^{\prime}$ with algebraic integers $\alpha=\gamma_{1} / 3$ and $\alpha^{\prime}=\gamma_{2} / 3$ conjugate over $K$ provided that $\gamma / 3 \in \mathbb{Z}_{K}$. The minimal polynomial for $\gamma / 3$ over $K$ is either $x^{3}+p x / 3-\ell / 27$, if $\ell=\gamma_{1} \gamma_{2} \gamma_{3}=\sqrt{\Delta} \in K$, or $\left(x^{3}+p x / 3\right)^{2}-\Delta / 729$, if $\ell=\sqrt{\Delta} \notin K$. In both cases, $\gamma / 3 \in \mathbb{Z}_{K}$ if and only if $\Delta / 729 \in \mathbb{Z}_{K}$.

The discriminant of the polynomial $x^{3}-2$ is equal to 108 . It is not divisible by 729. Nevertheless, $2^{1 / 3}$ is a difference of two conjugate integers, even units. One can check that $2^{1 / 3}$ is a difference of two roots of an irreducible over $\mathbb{Q}$ polynomial

$$
x^{18}-6 x^{15}+7 x^{12}+4 x^{9}+115 x^{6}+2 x^{3}+1 .
$$

This shows that the construction of $\alpha$ might be nontrivial even for cubic $\beta$. We now are in the position to state the main results of this paper. Below, $\Delta=-4 p^{3}-27 q^{2}$.

THEOREM 1. Let $K$ be a number field, and let $\beta$ be a cubic algebraic integer over $K$ whose minimal polynomial over $K$ is $x^{3}+p x+q$. If $p / 9 \in \mathbb{Z}_{K}$, then $\beta$ can be written as a difference of two conjugates over $K$ of an algebraic integer. Furthermore, the latter number can be chosen to be of degree 9 , if $\sqrt{\Delta} \in K$, and 18 otherwise.

Note that $\sqrt{\Delta} \in K$ if the Galois group of $K\left(\beta_{1}, \beta_{2}, \beta_{3}\right) / K$ is cyclic (of order 3), and $\sqrt{\Delta} \notin K$, if the Galois group is $S_{3}$ (of order 6).

THEOREM 2. Let $K$ be a number field, and let $e \geqslant 2$ be an integer. Assume that $\beta$ is an algebraic integer whose minimal polynomial over $K$ is of the form $f\left(x^{e}\right)$, where $f(x) \in \mathbb{Z}_{K}[x]$. Then $\beta$ can be written as a difference of two conjugates over $K$ of an algebraic integer.

In particular, if $x^{e}-q$, where $e \geqslant 2$, is irreducible over $\mathbb{Q}$, then $q^{1 / e}$ is a difference of conjugate algebraic integers. (The conditions on $q \in K$ under which the polynomial $x^{e}-q$ is irreducible over an arbitrary field $K$ can be found in [5, Chapter VIII, Section 9].)

COROLlaRY 2. If $e \geqslant 2$ is a positive integer, $q \in \mathbb{Z}$ and $q^{1 / e}$ has degree $e$ over $\mathbb{Q}$, then $q^{1 / e}$ can be written as a difference of two conjugates over $\mathbb{Q}$ of an algebraic integer. 


\section{Proof of Theorem 1}

Let $\gamma=\gamma_{1}=\beta_{1}-\beta_{3}$ be as in Section 2. Since $\gamma_{1}+\gamma_{3}+\gamma_{3}=0$,

$$
\gamma_{1} \gamma_{2}+\gamma_{1} \gamma_{3}+\gamma_{2} \gamma_{3}=3\left(\beta_{1} \beta_{2}+\beta_{1} \beta_{3}+\beta_{2} \beta_{3}\right)=3 p
$$

and $\gamma_{1} \gamma_{2} \gamma_{3}=\ell=\sqrt{\Delta}$, we deduce that $\gamma$ is a root of $x^{3}+3 p x-\ell=0$. The idea of the proof is to look at the action of the group $G$ of the Galois extension $K\left(\beta_{1}, \beta_{2}, \beta_{3}\right) / K$ on the number

$$
\alpha=\gamma / 3+(m-\ell / 27)^{1 / 3} \text {. }
$$

The Galois group $G$ is cyclic if and only if $\ell \in \mathbb{Z}_{K}$. Then $\gamma$ is of degree 3 over $K$. Otherwise, $G$ is $S_{3}$ and $\gamma$ is of degree 6 over $K$. Choose $m \in \mathbb{Z}$ such that $\alpha$ is of degree 9 or 18 over $K$, respectively. Clearly, $\alpha^{\prime}=\gamma_{2} / 3+(m-\ell / 27)^{1 / 3}$ is among the conjugates of $\alpha$ over $K$, so that $\alpha-\alpha^{\prime}=\left(\beta_{1}-\beta_{3}-\beta_{2}+\beta_{1}\right) / 3=\beta_{1}=\beta$. Since $\ell=\gamma^{3}+3 p \gamma$, the minimal polynomial of $\alpha$ over $K(\gamma)$ is

$$
x^{3}-\gamma x^{2}+\gamma^{2} x / 3+p \gamma / 9-m .
$$

Thus $\alpha$ is an algebraic integer if $\gamma^{2} / 3$ is an algebraic integer and, in addition to this, $p / 9 \in \mathbb{Z}_{K}$. We shall show that the second condition automatically implies the first one.

Note the minimal polynomial of $\gamma^{2} / 3$ over $K$ is

$$
x^{3}+2 p x^{2}+p^{2} x-\Delta / 27 \text {. }
$$

Indeed, this follows from the equality $\Delta=\ell^{2}=\left(\gamma^{3}+3 p \gamma\right)^{2}=27\left(\gamma^{2} / 3+p\right)^{2}\left(\gamma^{2} / 3\right)$ and from the fact that the numbers $\gamma_{1}^{2}, \gamma_{2}^{2}, \gamma_{3}^{2}$ are distinct. (Clearly, $\gamma_{1} \neq-\gamma_{2}$. Also, $\gamma_{1} \neq \gamma_{2}$, since, by [6, Lemma 1], the equality $2 \beta_{1}=\beta_{2}+\beta_{3}$ is impossible.) Notice that $\Delta / 27=-108(p / 9)^{3}-q^{2} \in \mathbb{Z}_{K}$, because $p / 9$ and $q$ are both in $\mathbb{Z}_{K}$. This completes the proof, since the condition $p / 9 \in \mathbb{Z}_{K}$ implies that $\gamma^{2} / 3$ is an algebraic integer.

REMARK. Formally, Lemma 5 of [6] is given for $K=\mathbb{Q}$ only, but the argument (map to the largest conjugate) remains the same for every $K$ which is a subfield of the field of complex numbers, including number fields. More generally, the equality $q_{1} \beta_{1}$ $=q_{2} \beta_{2}+\ldots q_{n} \beta_{n}$ with distinct algebraic numbers $\beta_{1}, \ldots, \beta_{n}, n \geqslant 3$, conjugate over an arbitrary field $K$ of characteristic 0 is impossible, if $q_{1}, \ldots, q_{n}$ are nonzero integers such that $\left|q_{1}\right| \geqslant\left|q_{2}\right|+\cdots+\left|q_{n}\right|$. See [2, Theorem 4], where such an argument could not be used, and thus an algebraic proof was given.

\section{Proof of Theorem 2}

If $e$ is even, then the theorem follows immediately, by our observation. Assume that $e$ is divisible by an odd prime $P$. Let $\varepsilon$ be the primitive $P$ th root of unity. We 
shall show first that there is a $\sigma \in G=G\left(K\left(\beta_{1}, \ldots, \beta_{d}\right) / K\right)$ such that $\sigma(\beta)=\beta \varepsilon$ and $\sigma(\varepsilon)=\varepsilon$.

Indeed, take an arbitrary $\sigma_{1} \in G$ which maps $\beta$ to its conjugate $\beta \varepsilon$. Assume that $\sigma_{1}(\varepsilon)=\varepsilon^{\ell}$, where $1<\ell \leqslant P-1$. Let $j$ be the smallest positive integer such that $\ell^{j} \equiv 1(\bmod P)$. Then $\sigma_{1}^{j} \operatorname{maps} \beta$ to $\beta \varepsilon^{(\ell+1)^{j-1}}$ and $\varepsilon$ to $\varepsilon$. It follows that we can take $\sigma$ to be a power of $\sigma_{1}^{j}$, if $\ell \neq P-1$. The alternative case, $\ell=P-1$, can only happen for $P>3$. Consider an automorphism $\sigma_{2} \in G$ which maps $\beta$ to its other conjugate $\beta \varepsilon^{2}$. If now $\sigma_{2}(\varepsilon)=\varepsilon^{s}$ with $s$ in the range $1 \leqslant s<P-1$, then we can apply the above argument. Alternatively, $\sigma_{2}(\varepsilon)=\varepsilon^{P-1}$, but then the automorphism $\sigma_{1} \sigma_{2}$ maps $\beta$ to $\beta \varepsilon^{P-1}$ and $\varepsilon$ to $\varepsilon$. Clearly, there is a power of $\sigma_{1} \sigma_{2}$, which maps $\beta$ to $\beta \varepsilon$ and $\varepsilon$ to $\varepsilon$.

Set

$$
\gamma=\gamma_{1}=\beta\left(P-1+(P-2) \varepsilon+(P-3) \varepsilon^{2}+\cdots+\varepsilon^{P-2}\right)=P \beta /(1-\varepsilon),
$$

and $\gamma_{i}=\sigma^{i}(\gamma)=\gamma \varepsilon^{i-1}$ for $i=2,3, \ldots, P$. Now, $\gamma_{1}, \ldots, \gamma_{P}$, are all roots of the polynomial $x^{P}-(P \beta /(1-\varepsilon))^{P}$. Assume that $\gamma$ is of degree $D$ over $K$. We choose $m \in \mathbb{Z}$ in such a way that

$$
\alpha=\gamma / P+\left(m-(\gamma / P)^{P}\right)^{1 / P}
$$

is of degree $P D$ over $K$. Then, as above, $\alpha^{\prime}=\gamma_{2} / P+\left(m-(\gamma / P)^{P}\right)^{1 / P}$ is conjugate to $\alpha$ over $K$ and $\alpha-\alpha^{\prime}=(\gamma-\sigma(\gamma)) / P=\beta$, using $\gamma / P=\beta /(1-\varepsilon)$. Also, $\alpha$ is a root of the polynomial

$$
x^{P}-\sum_{j=1}^{P-1}(-1)^{j}\left(\frac{\beta}{1-\varepsilon}\right)^{j}\left(\begin{array}{c}
P \\
j
\end{array}\right) x^{P-j}-m .
$$

It remains to prove that $\alpha$ is an algebraic integer. Clearly, so are $\beta$ and $m \in \mathbb{Z}$. Since every binomial coefficient is divisible by $P$, it suffices to show that $P /(1-\varepsilon)^{j}$ is an algebraic integer for every $j=1, \ldots, P-1$. This will be the case if $P /(1-\varepsilon)^{P-1}$ is an algebraic integer, because so is $1-\varepsilon$ and its natural powers. The product of the conjugates of $P /(1-\varepsilon)^{P-1}$ over $\mathbb{Q}$ is equal 1 , thus, equivalently, it suffices to show that $(1-\varepsilon)^{P-1} / P$ is an algebraic integer. This is exactly the case, because the coefficients of the polynomial

$$
h(x)=(1-x)^{P-1}-1-x-\cdots-x^{P-1}=\sum_{j=1}^{P-2}(-1)^{j}\left(\left(\begin{array}{c}
P-1 \\
j
\end{array}\right)+(-1)^{j-1}\right) x^{j}
$$

are all divisible by $P$ (check this for $j$ even and odd)! Since $h(\varepsilon) / P=(1-\varepsilon)^{P-1} / P$, and $\varepsilon$ itself is an algebraic integer, the proof is completed. 


\section{Similar QUESTIONS}

It is somewhat surprising that both questions are very easy to answer if we replace the word "difference" by one of the words "sum" or "product". In fact, every algebraic number $\beta$ is a sum of two distinct conjugates $\alpha$ and $\alpha^{\prime}$ over $K$. (Just take them both as roots of an irreducible over $K(\beta)$ polynomial $x^{2}-\beta x+m$ with nonzero $m \in \mathbb{Z}$.) Similarly, by taking $\alpha$ and $\alpha^{\prime}$ as roots of some irreducible over $K(\beta)$ polynomial $x^{2}+m x+\beta$, where $m \in \mathbb{Z}$, we see that every nonzero algebraic number $\beta$ is a product of two distinct conjugates $\alpha$ and $\alpha^{\prime}$ over $K$, whereas zero is only expressible as the product of two zeroes (see also [1, Section 3]). In both cases, we can positively answer to the second question, because the numbers $\alpha$ and $\alpha^{\prime}$ are algebraic integers provided that so is $\beta$.

With the word "difference" being replaced by the word "quotient", it was shown in [3] that a nonzero $\beta$ is equal to $\alpha / \alpha^{\prime}$ with some $\alpha$ and $\alpha^{\prime}$ conjugate over $K$ if and only if there is an automorphism $\sigma$ in the Galois group of $K\left(\beta_{1}, \ldots, \beta_{d}\right) / K$ of order $n$ such that $\prod_{i=0}^{n-1} \sigma^{i}(\beta)$ is a root of unity. Every such $\beta$ is also a quotient of two conjugates over $K$ of an algebraic integer. (Just write $\beta=\alpha / \alpha^{\prime}=(m \alpha) /\left(m \alpha^{\prime}\right)$ with some nonzero $m \in \mathbb{Z}$ such that $m \alpha$ is an algebraic integer.) In order to ask the "right" questions for the "quotient" case, we replace the words "algebraic integer" by the word "unit". Recall that $\beta$ is a unit if both $\beta$ and $1 / \beta$ are algebraic integers.

QUESTION 1'. Which numbers $\beta$ can be written as a quotient $\alpha / \alpha^{\prime}$ of two conjugates over $K$ of a unit?

QUESTION $2^{\prime}$. Is every unit which is expressible as a quotient of two conjugates over $K$ of an algebraic number also expressible as a quotient of two conjugates over $K$ of a unit?

The answer to Question $2^{\prime}$ is positive. This follows from the next theorem which also answers Question 1', because $\beta$ which are quotients of two units are units themselves.

THEOREM 3. Given a number field $K$, a unit $\beta$ is expressible as a quotient of two conjugates over $K$ of a unit if and only if there is an automorphism $\sigma$ in the Galois group of the normal extension of $K(\beta)$ over $K$ of order $n$ such that $\prod_{i=0}^{n-1} \sigma^{i}(\beta)$ is a root of unity.

ProOF: The necessity of the condition follows from [3, Theorem 1.1]. In order to prove that the condition is also sufficient, we set

$$
\gamma=\prod_{i=0}^{n-1}\left(\sigma^{i}(\beta)\right)^{n-i-1}
$$


Then $\gamma / \sigma(\gamma)=\beta^{n} / \theta$, where $\theta=\prod_{i=0}^{n-1} \sigma^{i}(\beta)$ is a root of unity. Among the conjugates of $\gamma^{1 / n}$ over $K$ there is at least one number of the form $\rho \sigma(\gamma)^{1 / n}$, where $\rho^{n}=1$. We have that $\gamma^{1 / n} /\left(\rho \sigma(\gamma)^{1 / n}\right)=\mu \beta$, where $\mu$ is a root of unity. Furthermore, $\gamma^{1 / n}$ is a unit, since so is $\beta$. It suffices to show that, given arbitrary, say $\ell$ th, root of unity $\mu$ and a unit $\beta$, which is a quotient of two conjugate over $K$ units $\alpha$ and $\alpha^{\prime}$, the number $\mu \beta$ is also expressible by a similar quotient.

Set $\delta=\alpha \omega$, where $\omega=\left(m+\sqrt{m^{2}-1}\right)^{1 / \ell}$. As in [3, Lemma 3.1], given arbitrary extension of $K$, say $L$, which contains the numbers $\alpha$ and $\alpha^{\prime}$, there is an $m \in \mathbb{Z}$ such that $\alpha$ and $\alpha^{\prime}$ are conjugate over $K(\omega)$ and the degree of $\omega$ over $L$ is $2 \ell$ (or, equivalently, the polynomial $1-2 m x^{\ell}+x^{2 \ell}$ is irreducible over $L$ ). Let $L$ be the normal closure of $K\left(\alpha, \mu_{\ell}\right)$ over $K$. Here, $\mu_{\ell}$ is the primitive $\ell$ th root of unity. Note that $L(\omega)$ is the Galois extension over $L$ and so it is over $K$, because $L / K$ is normal. Then there is an automorphism $\sigma$ of the Galois group of $L(\omega) / K$ such that $\sigma(\alpha)=\alpha^{\prime}$ and $\sigma(\omega)=\mu^{-1} \omega$. (We can take $\sigma$ as a composition of an automorphism taking $\omega$ to $\omega$ and $\alpha$ to $\alpha^{\prime}$ and an automorphism fixing $L$ and taking $\omega$ to $\mu^{-1} \omega$.) It follows that $\delta$ and $\sigma(\delta)$ are conjugate over $K$, and

$$
\frac{\delta}{\sigma(\delta)}=\frac{\alpha \omega}{\alpha^{\prime} \mu^{-1} \omega}=\frac{\mu \alpha}{\alpha^{\prime}}=\mu \beta
$$

Moreover, $\delta=\alpha \omega$ is a unit, because so are $\alpha$ and $\omega$.

\section{SPECULATIONS CONCERNING POSSIBLE GENERALISATION}

Let $\sigma$ be an automorphism in the Galois group of $K\left(\beta_{1}, \ldots, \beta_{d}\right) / K$ which maps $\left(\beta_{1}, \beta_{2}, \ldots \beta_{n}\right)$ to $\left(\beta_{n}, \beta_{1}, \ldots, \beta_{n-1}\right)$, where $\beta_{1}+\cdots+\beta_{n}=0$, and every $\beta_{i}$ with $i>n$ to some $\beta_{j}$ with $j=j(i)>n$. We know that only such $\beta$ are differences of two conjugates over $K$ of an algebraic number. Setting

$$
\gamma=(n-1) \beta_{1}+(n-2) \beta_{2}+\cdots+\beta_{n-1},
$$

we can simply choose $\alpha=\gamma / n$ and $\alpha^{\prime}=\sigma(\alpha)$ in order that $\alpha-\alpha^{\prime}=\beta$. Clearly, $\alpha$ already is an algebraic integer if so is $\gamma / n$. If $\alpha$ is not an algebraic integer, we can still obtain one from it by adding another algebraic number $\delta$ so that $\alpha+\delta$ is an algebraic integer and $N_{\alpha} \cap N_{\delta}=K$. (Given a field $K$ and an algebraic number $\alpha$, by $N_{\alpha}$ we denote the normal extension of $K(\alpha)$ over $K$.) This would immediately imply the positive answer to Question 2. Indeed, setting $G_{1}$ and $G_{2}$ for the Galois groups of $N_{\alpha} / K$ and $N_{\delta} / K$, respectively, we have that the Galois group of $N_{\alpha} N_{\delta} / K$ is $G_{1} \times G_{2}$ (see [5, Chapter VIII, Section 1]). In case if $K=\mathbb{Q}$ and $\alpha=\sqrt{1 / 2}$, we took 
$\delta=\sqrt{3 / 2}$. Then, the respective normal extensions are $N_{\alpha}=\mathbb{Q}(\sqrt{2}), N_{\delta}=\mathbb{Q}(\sqrt{6})$ whose intersection is $\mathbb{Q}(\sqrt{2}) \cap \mathbb{Q}(\sqrt{6})=\mathbb{Q}$.

However this is, in general, impossible. On applying the automorphisms which map $\alpha$ to their conjugates and $\delta$ to itself, we conclude that all differences between conjugates of $\alpha$ must be algebraic integers. In case if $K=\mathbb{Q}$ and $\beta_{1}=2^{1 / 3}, \beta_{2}=2^{1 / 3} \varepsilon$, $\beta_{3}=2^{1 / 3} \varepsilon^{2}$, where $\varepsilon$ is the complex root of $\varepsilon^{3}=1$, the Galois group of $N_{\beta} / \mathbb{Q}$ is $S_{3}$. The difference between $\alpha=\left(2 \beta_{1}+\beta_{2}\right) / 3$ and its conjugate $\left(2 \beta_{2}+\beta_{1}\right) / 3$ is not an algebraic integer, so that, in principle, it is impossible choose $\delta$, as required. We still managed to show that $2^{1 / 3}$ is a difference of two conjugate algebraic integers, using $\delta=\left(1-2\left(\varepsilon-\varepsilon^{2}\right) / 9\right)^{1 / 3}$ such that $\alpha+\delta$ is an algebraic integer and $N_{\alpha} \cap N_{\delta}=\mathbb{Q}(\sqrt{-3})$ is not too big.

The method described in Sections 3 and 4 can be generalised to algebraic numbers of arbitrary degree. If say $\beta$ is of degree $d=4$, and the 4-cycle (1234) belongs to the Galois group of $N_{\beta} / K$, we can set $\gamma=\gamma_{1}=3 \beta_{1}+2 \beta_{2}+\beta_{3}$ and, using $\gamma_{1}+\cdots+\gamma_{4}=0$, compute $r^{\prime}, p^{\prime}$ and $q^{\prime}$ such that $\gamma_{1}, \ldots, \gamma_{4}$ are all roots of the polynomial $x^{4}+r^{\prime} x^{2}$ $+p^{\prime} x+q^{\prime}$. Here, $r^{\prime}, p^{\prime}, q^{\prime}$ depend on the coefficients $r, p, q$ of the minimal polynomial for $\beta$, say $x^{4}+r x^{2}+p x+q$. Setting

$$
\alpha=\gamma / 4+\left(m-q^{\prime} / 256\right)^{1 / 4}
$$

with appropriate $m \in \mathbb{Z}$, we see that $\beta$ is a difference of $\alpha$ and its conjugate $\alpha^{\prime}=\gamma_{2} / 4+\left(m-q^{\prime} / 256\right)^{1 / 4}$. Now, $\alpha$ is a root of the polynomial

$$
x^{4}-\gamma x^{3}+3 \gamma^{2} x^{2} / 8-\gamma^{3} x / 16-m-\left(r^{\prime} \gamma^{2}+p^{\prime} \gamma\right) / 256
$$

It is not difficult to see that we shall get some advantage by demanding that $3 \gamma^{2} / 8$, $\gamma^{3} / 16$ and $\left(r^{\prime} \gamma^{2}+p^{\prime} \gamma\right) / 256$ are all algebraic integers compared to the trivial method (in which we ask the number $\gamma / 4$ to be an algebraic integer). This is however too technical for this paper.

At first glance, it may seem that the condition $p / 9 \in \mathbb{Z}_{K}$ may be easily replaced by the weaker condition $p / 3 \in \mathbb{Z}_{K}$. A cubic which is the root of the polynomial $x^{3}+p x+q$, by the Cardano formulae, is equal to say $\zeta+\omega$, where

$$
\zeta^{3}=-q / 2+\sqrt{p^{3} / 27+q^{2} / 4}, \quad \omega^{3}=-q / 2-\sqrt{p^{3} / 27+q^{2} / 4} .
$$

Both $\zeta$ and $\omega$ are roots of the equation $x^{6}+q x^{3}-(p / 3)^{3}=0$. Assume, for simplicity, that the polynomial $x^{6}+q x^{3}-(p / 3)^{3}$ is irreducible over $K$. If $p / 3 \in \mathbb{Z}_{K}$, then $\zeta$ and $\omega$ are both algebraic integers. Furthermore, by Theorem $2, \zeta=\alpha-\alpha^{\prime}$ and $\omega=\gamma-\gamma^{\prime}$, where $\alpha$ and $\gamma$ are algebraic integers, $\alpha$ and $\alpha^{\prime}$ are conjugate over $K$ (and so are $\gamma$ and $\left.\gamma^{\prime}\right)$. Assume that $L=K\left(\varepsilon, \sqrt{p^{3} / 27+q^{2} / 4}\right)$ is of maximal degree 4 over $K$. Then, 
as in Theorem 2, $\alpha$ and $\gamma$ can be chosen to be of degree 9 over $L$, and thus of degree 36 over $K$. Clearly, $\alpha+\gamma$ and $\alpha^{\prime}+\gamma^{\prime}$ are conjugate over $K$ if they are of maximal degree 324 over $K$. However, if $\alpha$ and $\gamma$ are chosen as in Theorem 2, the degree of $\alpha+\gamma$ is only 108 (which is too small)! One has to be cautious in constructing examples via sums: for instance, $\sqrt{2}+\sqrt{6}$ and $\sqrt{3}$ are both differences of two conjugates over $\mathbb{Q}$ of algebraic integers (see Section 1 and Observation), but their sum, $\sqrt{2}+\sqrt{3}+\sqrt{6}$, is not expressible by a difference of two conjugates over $\mathbb{Q}$ (see [3]).

\section{REFERENCES}

[1] A. Dubickas, 'The Remak height for units', Acta Math. Hungar. (to appear).

[2] A. Dubickas, 'On the degree of a linear form in conjugates of an algebraic number', Illinois J. Math. (to appear).

[3] A. Dubickas and C.J. Smyth, 'Variations on the theme of Hilbert's Theorem 90', Glasgow Math. J. (to appear).

[4] D. Hilbert, 'Die Theorie der algebraischen Zahlkörper', Jahresber. Deutsch. Math.-Verein 4 (1897), 175-546. English translation by I.T. Adamson: The theory of algebraic number fields, (Springer-Verlag, Berlin, 1998).

[5] S. Lang, Algebra (Addison-Wesley Publishing, Reading, Mass., 1965).

[6] C.J. Smyth, 'Conjugate algebraic numbers on conics', Acta Arith. 40 (1982), 333-346.

Department of Mathematics and Informatics

Vilnius University

Naugarduko 24

Vilnius 2600

Lithuania

e-mail: arturas.dubickas@maf.vu.lt 